\title{
Residual Disease Activity and Associated Factors in Psoriatic Arthritis
}

\author{
Ennio Lubrano ${ }^{1} \mathbb{D}$, Silvia Scriffignano ${ }^{1}$, and Fabio Massimo Perrotta ${ }^{1}$ (D)
}

ABSTRACT. Objective. Remission or low disease activity should be the treatment target of psoriatic arthritis (PsA). However, residual disease activity (RDA) in some domains could persist. The aim of this study was to assess RDA and its associated factors in a group of patients with PsA.

Methods. Patients with PsA were enrolled if they satisfied ClASsification for Psoriatic ARthritis (CASPAR) criteria with $>6$ months' followup and achieved a status of low disease activity (LDA), minimal disease activity (MDA), or remission [Disease Activity Index for PsA (DAPSA) remission or very low disease activity (VLDA)]. RDA was assessed by the percentage of patients who had, although in LDA or remission, tender and/or swollen joints > 1, Leeds Enthesitis Index > 1, Health Assessment Questionnaire > 0.5 , Psoriasis Area Severity Index (PASI) $>1$, patient's global assessment $>20$, physician visual analog scale $($ VAS $)>20$, and VAS pain $>15$. Associated factors of RDA were also assessed.

Results. Of 113 enrolled patients, 78 (69\%) were in MDA. Moreover, DAPSA remission was observed in 46 $(40.7 \%)$ while VLDA only in 32 (28.3\%) of patients with PsA. VLDA seems to be the most stringent criterion, with a minimal RDA only in the VAS physician in 1 patient $(3.1 \%)$ and none in the different domains, while patients in MDA had RDA in tender joints (14.1\%), VAS pain (29.4\%) and PASI $>1$ or body surface area $(\mathrm{BSA})>3 \%(17.9 \%)$. Of note, although patients in DAPSA remission show a very low rate of RDA in almost all domains, 12 (26\%) of them show a PASI > 1 or BSA > 3\%. Finally, LDA shows RDA in higher percentages, mainly in patient-reported outcomes, tender joints, and skin domain.

Conclusion. RDA can be recognized in patients with PsA. VLDA seems to be the most stringent composite index to identify patients in the absence of RDA.

Key Indexing Terms: composite indices, psoriatic arthritis, remission, residual disease activity

Psoriatic arthritis (PsA) is a multifaceted chronic inflammatory disease characterized by an association of psoriasis and arthritis ${ }^{1}$. It can be recognized as a "syndrome" in which different manifestations (arthritis, skin involvement, enthesitis, extraarticular involvement, and comorbidities) "run together." In the context of the disease, there are still some unmet needs that should be addressed, mainly on treatment strategies ${ }^{2}$. The achievement of the best possible disease control such as disease remission or low disease activity (LDA) have been proposed as treatment targets and may be achievable goals for patients with PsA ${ }^{3,4,5}$. The recent treat-to-target recommendation stated that remission or LDA should be the target of treatment ${ }^{6}$. However, because of the complexity of the disease, unidimensional and multidimensional disease activity indices that encompass different disease domains were developed. Of these, the Disease Activity Index for PsA

${ }^{1}$ E. Lubrano, MD, PhD, Associate Professor of Rheumatology, S. Scriffignano, MD, Specialist Registrar in Rheumatology, F.M. Perrotta, MD, PhD,

Lecturer in Physical Medicine and Rehabilitation, Dipartimento di Medicina e Scienze della Salute "Vincenzo Tiberio", Università degli Studi del Molise, Campobasso, Italy.

Address correspondence to Prof. E. Lubrano, Head of Academic Rheumatology Unit and MoRhe Project, Dipartimento di Medicina e Scienze della Salute

"Vincenzo Tiberio," Università degli Studi del Molise, Via Giovanni Paolo II, C/da Tappino,86100 Campobasso, Italy.Email:enniolubrano@hotmail.com.

Accepted for publication October 16, 2019.
(DAPSA) and the minimal disease activity (MDA) criteria have been included as treatment targets in the recent recommendation ${ }^{6,7,8}$. DAPSA is based on the assessment of tender and swollen joints, pain, patient's global assessment (PtGA) of disease activity, and C-reactive protein (CRP; mg/dl). A DAPSA $\leq 4$ defines a status of remission while a DAPSA $\leq 14$ a status of $\mathrm{LDA}^{7}$. Patients are considered in MDA when they satisfy $5 / 7$ of previously published criteria ${ }^{8,9}$. Disease control with the lowest grade of disease activity is important because achieving sustained MDA (defined as MDA for over 12 mos at consecutive clinic visits) reduced radiographic joint damage progression over a 3 -year period, as shown by Coates, et $a l^{10}$. In a more recent study, the same authors proposed a more stringent definition of remission (very low disease activity; VLDA) in which all $7 / 7$ criteria had to be satisfied ${ }^{11}$. However, owing to the construction of these indices, residual disease activity (RDA) could persist, mainly in patients who achieved less stringent criteria such as LDA or MDA. At present, a few reports are available on RDA in PsA patients when they achieve a status of remission or $\mathrm{LDA}^{12}$. Moreover, it is possible that the effect of RDA on PsA and its consequences on the management of this condition might be an important issue not only for physicians, but mainly for patients. The aim of our study was to investigate RDA in the different PsA domains (articular, skin, enthesis), systemic 
inflammation (CRP), and patient-reported outcomes (PRO), in patients in DAPSA LDA, MDA, or remission (VLDA, DAPSA $\leq 4)$. A secondary endpoint was to analyze the differences between PsA patients with or without RDA in each single domain, to evaluate factors associated to RDA.

\section{MATERIALS AND METHODS}

Patient selection. In this cross-sectional analysis of a longitudinal cohort, patients were enrolled at the Rheumatology Unit, Department of Medicine and Health Science - University of Molise. During the period January 1, 2017, to December 31, 2018, all patients with PsA who were taking at least a 6-month followup treatment with conventional (cs) and biological (b) disease-modifying antirheumatic drugs (DMARD) were considered potentially eligible for the study.

We used the following inclusion criteria:

(1) PsA classified with the ClASsification criteria for Psoriatic ARthritis (CASPAR),

(2) age $>18$ years,

(3) at least 6 months' followup at the study visit,

(4) stable treatment with a csDMARD or bDMARD for at least 6 months,

(5) in a condition of LDA (DAPSA score $\leq 14$ ), remission (DAPSA $\leq 4$ ), MDA, or VLDA.

The study protocol was in compliance with the Declaration of Helsinki and written consent was obtained from each participant. The study was approved by the Institutional Review Board of the University of Molise (protocol n. 0001-09-2017).

Data collection. Patient data collection included medical history, physical examination, current use of medications, and laboratory assessment. Demographics and disease characteristics including age, sex, disease duration, and pattern of articular manifestation were taken into account. The clinical assessment encompassed the number of tender joints (of the 68 assessed joints) and swollen joints (total of 66 joints), enthesitis, and dactylitis. Enthesitis was measured using the Leeds Enthesitis Index (LEI) ${ }^{13}$, and dactylitis as present/absent. Skin assessment included the Psoriasis Area Severity Index (PASI) score and the body surface area (BSA) ${ }^{14}$. The Health Assessment Questionnaire (HAQ) ${ }^{15}$ was used to assess function. PtGA and pain assessment on visual analog scale (VAS) were performed by all patients. Physician's global evaluation of disease activity on a VAS scale was also recorded ${ }^{16}$. CRP was also collected.

$M D A, V L D A, D A P S A$ remission, and DAPSA LDA. MDA was defined according to Coates, $e t a l^{8}$. Patients were considered in MDA when they satisfied $5 / 7$ of the following criteria: tender joint count $\leq 1$; swollen joint count $\leq 1$; PASI $\leq 1$ or BSA $\leq 3 \%$; patient pain VAS score $\leq 15$; patient global disease activity VAS score $\leq 20$; HAQ score $\leq 0.5$; and tender entheseal points $\leq 1^{17}$. Moreover, MDA 6/7, MDA joints, MDA joint/skin, and MDA skin were also analyzed ${ }^{12}$.

VLDA was satisfied when all 7 criteria were met ${ }^{11}$. DAPSA score was identified according to Schoels, et al and was calculated by adding the number of tender and swollen joints, VAS pain, PtGA, and CRP (mg/dl) ${ }^{7}$. DAPSA score $\leq 4$ identified remission while DAPSA $\leq 14$ was a condition of LDA.

Assessment of RDA. RDA was assessed by the percentage of patients who had, although in DAPSA LDA, MDA, VLDA, or DAPSA remission, tender and/or swollen joints > 1, LEI > 1, HAQ > 0.5, PASI > 1, PtGA > $20 \mathrm{~mm}$, and VAS pain $>15 \mathrm{~mm}^{17}$. PsA patients with RDA in the different domains were compared to patients without RDA to identify factors associated with RDA. HAQ is considered a measure of function and therefore we evaluated the rate of patients with a $\mathrm{HAQ}>0.5$ because it is part of the MDA criteria.

Finally, the physician's global assessment (PGA) of disease activity was also performed, in the same fashion of a previous study and expressed as VAS $\leq 20$, meaning a status of good control of the disease or remission/ LDA. This was considered the external anchor for the assessment of remis$\operatorname{sion}^{16}$. Therefore, we aimed to evaluate any concordance with MDA, VLDA, DAPSA remission, and LDA with the external anchor.

Statistical analysis. Proportions of patients achieving each definition of LDA and remission were calculated. The proportion of RDA was established for clinical domains of PsA (articular, enthesitis, psoriasis), HAQ, VAS pain, PtGA, and PGA of disease activity, and levels of CRP. Normal distribution was assessed by using the D'Agostino-Pearson's test. Categorical variables were analyzed by chi-square test with Yates' correction or Fisher's exact test. The significance of the differences was determined using the Mann-Whitney $U$ test for unpaired samples. Factors associated with RDA in each domain were studied by evaluating the differences between PsA patients with or without RDA in each single domain using Mann-Whitney for categorical variables or Fisher's exact test for non-categoricalones. Resultswereexpressedasmedian(interquartilerange). Concordance was assessed using Cohen's $\mathrm{k}$ coefficient and was considered as follows: < 0.20 poor; 0.21-0.40 fair; 0.41-0.60 moderate; 0.61-0.80 good; $0.81-1.00$ very good. All statistical procedures were 2 -sided; a significance level was accepted at $\mathrm{p}<0.05$.

\section{RESULTS}

Patient characteristics and overall disease activity. In the study period, 113 patients with PsA satisfied the inclusion criteria and were enrolled. All patients were in stable treatment with csDMARD or bDMARD (32 with etanercept, 23 with adalimumab, 13 with golimumab, 5 with ustekinumab, 16 with secukinumab, 2 with ixekizumab, and 22 with csDMARD monotherapy). All patients were in LDA defined as DAPSA $\leq 14$. The main clinical characteristics of enrolled patients are shown in Table 1.

Achievement of MDA, MDA 6/7, MDA joints, MDA joint/skin, $M D A$ skin, DAPSA remission, and VLDA. Figure 1 describes the achievement of all targets of enrolled patients with PsA, as well as the percentage of global disease activity assessed by physicians (VAS physician $\leq 20$ ). In particular, 76 patients with PsA $(67.2 \%)$ were deemed as VAS $<20$ by the physicians.

$R D A$ and associated factors. Overall, 81 patients (71.7\%) showed $\mathrm{RDA}$ in at least 1 domain. Further, the rate of patients with RDA was significantly higher among csDMARD-treated patients compared to bDMARD-treated patients $(86.3 \%$ vs $63.7 \%, \mathrm{p}=$ 0.04). Figure 2 shows the rate of RDA in the different disease domains observed. According to these results, VLDA seems to be the most stringent criteria, with a minimal RDA only in the VAS physician in 1 patient (3.1\%) and none in the different domains, while patients in MDA had RDA in tender joints (11 patients, 14.1\%), VAS pain (23 patients, 29.4\%), and skin (14 patients, $17.9 \%)$. Of note, although patients in DAPSA remission show a very low rate of RDA in almost all domains, 12 (26\%) of them show a PASI > 1. Finally, DAPSA LDA patients show RDA with a higher percentage, mainly in PRO, tender joints, and skin domain (Figure 2). When the RDA was evaluated in those patients with physician's VAS $\leq 20$, the main domains involved were tender joints, $\mathrm{PRO}$, and skin (Figure 2).

Table 2A shows the comparison of different features between

Personal non-commercial use only. The Journal of Rheumatology Copyright $\subset$ 2020. All rights reserved. 
Table 1. Demographic and clinical disease activity characteristics of patients with PsA in LDA.

\begin{tabular}{lc}
\hline Characteristics & Values \\
\hline Female/male, $\mathrm{n}$ & $49 / 64$ \\
Mean age (SD), yrs & $53.7(12.4)$ \\
Disease duration, mean (SD), yrs & $8(8.8)$ \\
Axial involvement, $\mathrm{n}(\%)$ & $42(37.1)$ \\
Tender joints, median (IQR) & $1(0-2)$ \\
Swollen joints, median (IQR) & $0(0-1)$ \\
BSA, \% (IQR) & $1(1-3)$ \\
PASI, median (IQR) & $0.3(0-1)$ \\
Enthesitis (LEI), median (IQR) & $0(0-0)$ \\
CRP, mg/dl, median (IQR) & $0.3(0.2-0.4)$ \\
MDA 5/7, n (\%) & $78(69)$ \\
VLDA, $\mathrm{n}$ (\%) & $32(28.3)$ \\
DAPSA remission & $46(40.7)$ \\
HAQ, median (IQR) & $0.25(0.125-0.5)$ \\
VAS pain, median (IQR) & $16.5(10-30)$ \\
PtGA, median (IQR) & $20(10-30)$ \\
VAS physician, median (IQR) & $15(10-23.5)$ \\
Treatment, n (\%) & \\
csDMARD monotherapy & $22(19.4)$ \\
Etanercept & $32(28.3)$ \\
Adalimumab & $23(20.3)$ \\
Golimumab & $13(11.5)$ \\
Ustekinumab & $5(4.4)$ \\
Secukinumab & $16(14.1)$ \\
Ixekizumab & $2(1.7)$ \\
\hline PA. & \\
\hline
\end{tabular}

PsA: psoriatic arthritis; LDA: low disease activity; IQR: interquartile range; BSA: body surace area; LEI: Leeds Enthesitis Index; PASI: Psoriasis Area Severity Index; CRP: C-reactive protein; MDA: minimal disease activity; VLDA: very low disease activity; DAPSA: Disease Activity Index for Psoriatic Arthritis; HAQ: Health Assessment Questionnaire; VAS: visual analog scale; PtGA: patient's global assessment; csDMARD: conventional synthetic disease-modifying antirheumatic drugs.

PsA patients (all in DAPSA LDA as per protocol) with and without RDA in 3 different domains (articular, PRO, and skin). A more detailed comparison of patients with and without RDA in each domain is shown in Supplementary Tables $1 \mathrm{~A}$ and $1 \mathrm{~B}$ (available from the authors on request).

PsA patients with RDA in an articular domain (tender joints $>1$ ) had a significantly higher number of swollen joints, higher HAQ score, and higher values of VAS pain, PtGA, and physician VAS. Patients with RDA in PRO had significantly higher CRP values, HAQ, tender joints, and LEI. No factors were associated with RDA in skin domain, while reduced function (HAQ $>0.5$ ) was seen in patients with higher disease duration, CRP values, PASI values, pain, and PtGA values, when compared to non-categorical variables (Table 2, and Supplementary Table $1 \mathrm{~A}$, available from the authors on request).

Further, HAQ > 0.5 was associated with female sex and presence of axial involvement when compared to categorical variables (Supplementary Table 1B, available from the authors on request).
Concordance between PGA and MDA,DAPSA remission, and $V L D A$. When the physician's assessment $(\leq 20)$ was evaluated, a good agreement was found with MDA, while a fair agreement was found with DAPSA. Poor agreement was found with VLDA (Table 3).

\section{DISCUSSION}

The assessment of a complex disease such as PsA could be a difficult task most of the time. In fact, the development of composite indices in the last 10 years has tried to identify those domains that are important for physicians and patients, to set up a treat-to-target strategy.

The emerging factors associated with these indices are the identification of a primary target to be adopted in a treat-to-target strategy ${ }^{18}$, and secondarily, whether they are capable of reflecting the control of all domains. The latter has as a consequence the possibility that some RDA could persist even when patients are identified in a condition of LDA, MDA, VLDA, or remission. It is implicit that a condition of LDA or MDA could show RDA because of the intrinsic concept of LDA that it is not, per se, considered as a full control of the disease ${ }^{19}$. Therefore, the assessment of RDA could be an important aspect for the management of PsA, because LDA and MDA are widely used in routine clinical care as main treatment targets.

Our present study showed that RDA is detectable in PsA patients in a status of LDA, MDA, and even DAPSA remission, while VLDA seems to be the only index capable of identifying a condition of remission without RDA. To support this result, all patients in VLDA but one were deemed by the physician to have a VAS $\leq 20$ even if VLDA does not encompass the VAS PGA. In particular, our results showed that patients with PsA achieving a VLDA status did not have any residual raised CRP, demonstrating that no systemic inflammation was still present in those patients. Coates, et al showed in a recent study that, in their group of patients with PsA, a residual CRP was numerically lower in patients in remission, and concluded that the inclusion of CRP may be not necessary because of the absence of any effect on the achievement of remission or LDA or a HAQ score ${ }^{18}$. When RDA was evaluated as residual functional impairment (HAQ > 0.5), this was observed in a small percentage of MDA and DAPSA remission patients with PsA, while it was higher in those in LDA. These results are in keeping with a study performed recently in Turkey ${ }^{20}$, showing that the disease burden could still persist in some patients.

When the RDA to a condition of MDA and LDA was assessed, our results showed that all domains were involved, while the residual skin component was quite high in those achieving DAPSA remission, confirming its unidimensional capacity to assess mainly joint disease activity. This latter result could be deemed as good when patients attending rheumatology clinics are mainly "joint-focused" and skin is not an important aspect of the disease ${ }^{21}$. On the other hand, the median BSA was $1 \%$, showing a minimal skin involvement in our patients.

Personal non-commercial use only. The Journal of Rheumatology Copyright @ 2020 . All rights reserved. 


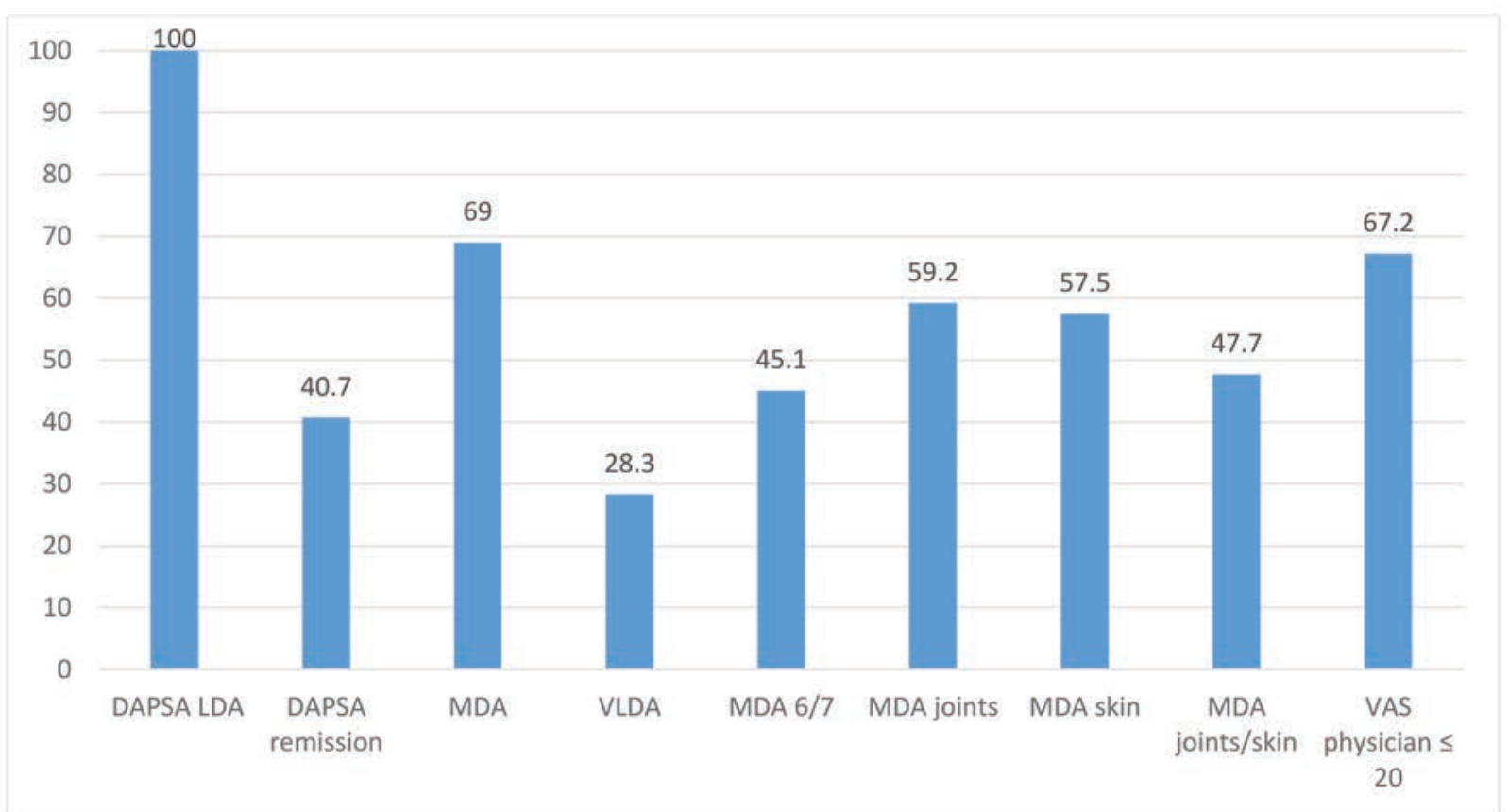

Figure 1. Percentage of PsA patients $(\mathrm{n}=113)$ in remission or low disease activity according to various indices. PsA: psoriatic arthritis; DAPSA: Disease Activity Index for Psoriatic Arthritis; LDA: low disease activity; MDA: minimal disease activity; VLDA: very low disease activity; VAS: visual analog scale.

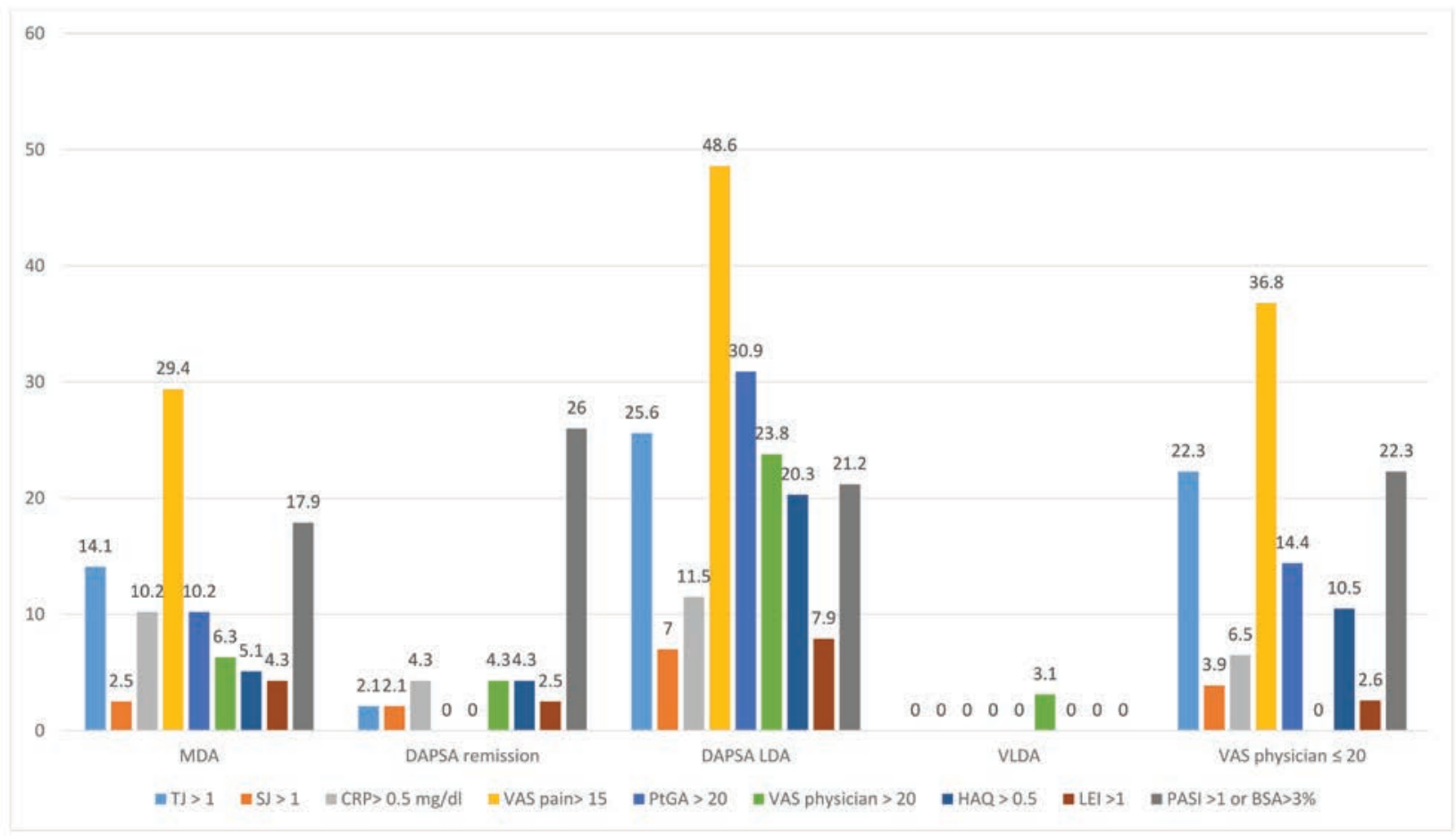

Figure 2. Residual disease activity in different domains according to the various indices used. MDA: minimal disease activity; DAPSA: Disease Activity Index for Psoriatic Arthritis; LDA: low disease activity; VLDA: very LDA; VAS: visual analog scale; TJ: tender joints; SJ: swollen joints; CRP: C-reactive protein; PtGA: patient's global assessment; HAQ: Health Assessment Questionnaire; LEI: Leeds Enthesitis Index; PASI: Psoriasis Area Severity Index; BSA: body surface area.

Personal non-commercial use only. The Journal of Rheumatology Copyright @ 2020 . All rights reserved.

Lubrano, et al: Residual disease activity in Ps $A$ 
Table 2A. Comparison of different features between PsA patients in DAPSA LDA with and without RDA in the articular domain (tender joint), PRO (VAS pain and PtGA), and skin domain (PASI; t test or Mann-Whitney $U$ test for unpaired samples; non-categorical variables).

\begin{tabular}{|c|c|c|c|c|c|c|c|c|c|c|c|c|}
\hline \multicolumn{13}{|c|}{ Disease Domains (Articular, PRO, and Skin) } \\
\hline & \multicolumn{3}{|c|}{ Tender Joints } & \multicolumn{2}{|c|}{ VAS Pain, mm } & \multicolumn{2}{|c|}{ PtGA, mm } & \multirow[b]{2}{*}{ RDA- } & \multirow[b]{2}{*}{$\mathrm{p}$} & \multicolumn{3}{|c|}{ PASI } \\
\hline & $\mathrm{RDA}+{ }^{\S}$ & RDA- & $\mathrm{p}$ & $\mathrm{RDA}^{\#}$ & RDA- & $\mathrm{p}$ & $\mathrm{RDA}+^{\circ}$ & & & $\mathrm{RDA}^{\$}{ }^{\$}$ & RDA- & $\mathrm{p}$ \\
\hline \multicolumn{13}{|c|}{ CRP mg/dl (median/ } \\
\hline IQR) & $0.3(0.2-0.48)$ & $0.2(0.2-0.39)$ & NS & $0.3(0.2-0.5)$ & $0.2(0.2-0.3)$ & $<0.01$ & $0.3(0.17-0.5)$ & $0.2(0.2-0.4)$ & NS & $0.2(0.2-0.4)$ & $0.3(0.2-0.4)$ & NS \\
\hline \multicolumn{13}{|c|}{ Tender joints (median/ } \\
\hline $\mathrm{IQR})$ & - & - & - & $1(0-2)$ & $0(0-1)$ & $<0.01$ & $1(0-3)$ & $1(0-1)$ & 0.05 & $0(0-2)$ & $1(0-2)$ & NS \\
\hline \multicolumn{13}{|c|}{ HAQ (median/ } \\
\hline $\mathrm{IQR})$ & $0.5(0.25-0.75)$ & $0.25(0-0.5)$ & $<0.01$ & $0.5(0.25-0.75)$ & $0.12(0-0.31)$ & $<0.01$ & $0.68(0.5-0.96)$ & $0.25(0-0.43)$ & $<0.01$ & $0.25(0-0.5)$ & $0.25(0.12-0.53)$ & NS \\
\hline \multicolumn{13}{|c|}{ LEI (median/ } \\
\hline IQR) & $0(0-1)$ & $0(0-0)$ & NS & $0(0-1)$ & $0(0-0)$ & $<0.01$ & $0(0-1)$ & $0(0-0)$ & $<0.01$ & $0(0-1)$ & $0(0-0)$ & NS \\
\hline VAS pain & $20(15-36)$ & $15(2.7-30)$ & $<0.01$ & - & - & - & $35(30-50)$ & $10(0-20)$ & $<0.01$ & $10(0-25)$ & $10(0-20)$ & NS \\
\hline PtGA & $25(20-32)$ & $15(10-29)$ & $<0.01$ & $28(20-40)$ & $10(0-10)$ & $<0.01$ & - & - & - & $17.5(0-25)$ & $20(10-30)$ & NS \\
\hline
\end{tabular}

Note: RDA in articular domain (swollen joints) and enthesitis (LEI) was not evaluated because of a small number of patients with RDA in these domains. ${ }^{\S}$ Tender joints RDA+: > 1. "VAS pain RDA+: > $15 \mathrm{~mm} .{ }^{\circ} \mathrm{PtGA}$ RDA+: > $20 \mathrm{~mm} .{ }^{\$} \mathrm{PASI}$ RDA+: > 1 . Values are median (IQR).

Table 2B. Comparison of different clinical features between PsA patients ( $\mathrm{n}$ ) in DAPSA LDA with and without RDA in the articular domain (tender joint), PRO (VAS pain and PtGA), and skin domain (PASI; Fisher's exact test; categorical variables).

\begin{tabular}{|c|c|c|c|c|c|c|c|c|c|c|c|c|}
\hline & \multicolumn{2}{|c|}{ Tender Joints } & \multicolumn{5}{|c|}{ VAS Pain, mm } & \multicolumn{2}{|l|}{ PtGA, mm } & \multirow{2}{*}{$\begin{array}{l}\text { PASI } \\
\text { RDA }^{\$}\end{array}$} & \multirow[b]{2}{*}{ RDA- } & \multirow[b]{2}{*}{$\mathrm{p}$} \\
\hline & $\mathrm{RDA}^{\S}$ & RDA- & $\mathrm{p}$ & $\mathrm{RDA}^{*}{ }^{\#}$ & RDA- & $\mathrm{p}$ & $\mathrm{RDA}+{ }^{\circ}$ & RDA- & $\mathrm{p}$ & & & \\
\hline Male & 16 & 46 & NS & 28 & 34 & NS & 16 & 46 & 0.04 & 21 & 40 & 0.01 \\
\hline $\begin{array}{l}\text { Axial } \\
\text { involvement }\end{array}$ & 2 & 39 & NS & 25 & 16 & NS & 16 & 25 & NS & 6 & 33 & NS \\
\hline $\begin{array}{l}\text { No axial } \\
\text { involvement }\end{array}$ & 6 & 56 & & 20 & 42 & & 19 & 43 & & 17 & 44 & \\
\hline
\end{tabular}

$\S$ Tender joints RDA+: > 1. ${ }^{*}$ VAS pain RDA+: > $15 \mathrm{~mm} .{ }^{\circ}$ PtGA RDA+: > $20 \mathrm{~mm} .{ }^{\$}$ PASI RDA+: > 1. RDA: residual disease activity; PsA: psoriatic arthritis; DAPSA: Disease Activity Index for Psoriatic Arthritis; LDA: low disease activity; PASI: Psoriasis Area Severity Index; PRO: patient-reported outcome; PtGA: patient's global assessment; VAS: visual analog scale; CRP: C-reactive protein; HAQ: Health Assessment Questionnaire; LEI: Leeds Enthesitis Index; IQR: interquartile range; NS: not significant.

Table 3. Concordance (Cohen's k) between physician's global assessment and the 3 definitions of remission and minimal disease activity indices evaluated.

\begin{tabular}{lcccc}
\hline & VAS Physician $\leq 20$ & DAPSA Remission & MDA & VLDA \\
\hline VAS physician $\leq 20$ & - & 0.24 & 0.64 & 0.15 \\
DAPSA remission & 0.24 & - & 0.35 & 0.57 \\
MDA & 0.64 & 0.35 & - & 0.31 \\
VLDA & 0.15 & 0.57 & 0.31 & - \\
\hline
\end{tabular}

DAPSA: Disease Activity Index for Psoriatic Arthritis; MDA: minimal disease activity; VLDA: very low disease activity; VAS: visual analog scale.

Moreover, when evaluating the factors associated to a potential RDA, our results showed that PsA patients in LDA with tender joints as RDA had a significantly higher number of swollen joints, higher values of VAS pain, PtGA, and physician VAS, as well as a higher HAQ score. This result implies that these patients have potentially more severe disease, and the physicians should pay more attention in the treatment strategy ${ }^{22}$. In fact, the RDA in tender joints could be deemed by patients as the trigger for a treatment change.
The discordance observed between the physician's judgment and the possibility of any RDA, mainly on tender and swollen joints, is in keeping with other studies ${ }^{23}$ and confirms, to a certain extent, that a true agreement between patients and physician's assessment is still an unmet need ${ }^{2}$.

Patients with RDA in PRO had significantly higher CRP values, higher $\mathrm{HAQ}$, tender joints, and LEI. This result could suggest that persistence of systemic inflammation, loss of function, and some clinical manifestations are definitely perceived as 
persistence of disease activity by the patients and deemed as not a complete disease control.

No factors were associated with RDA in the skin domain, while reduced function $(\mathrm{HAQ}>0.5)$ was associated with female sex and presence of axial involvement. Patients with residual systemic inflammation (CRP $>0.5 \mathrm{mg} / \mathrm{dl}$ ) had significantly higher median tender joints, LEI, pain, and PtGA as well as higher PASI. Another interesting result is the higher rate of RDA in patients treated with csDMARD compared to bDMARD. This could be due to a better effectiveness of these drugs in all disease domains.

Our study has strengths as well as limitations. We decided to perform an assessment in a group of patients in stable treatment and in LDA only with a cross-sectional design, aiming to get an overview of the RDA. At the same time, we did not perform any analysis on potential treatment implications in those patients in RDA (such as change therapy) owing to the study design, and this aspect could be of some interest for practical issues. However, our study tried to identify potential factors associated with an RDA condition, and as far as we know, this is a novelty in this intriguing topic. The results of our study could be useful to identify patients in which some RDA are potential factors driving a possible change of treatment strategy, even if the same patients achieved a condition such as LDA or MDA.

RDA could be recognized in patients with PsA, and this seems more present when some targets are identified for the assessment of disease activity. VLDA seems to be the most stringent composite index to identify patients in absence of RDA.

\section{REFERENCES}

1. Ritchlin CT, Colbert RA, Gladman DD. Psoriatic arthritis. N Engl J Med 2017;376:957-70.

2. Helliwell P, Coates L, Chandran V, Gladman D, de Wit M, FitzGerald O, et al. Qualifying unmet needs and improving standards of care in psoriatic arthritis. Arthritis Care Res 2014;66:1759-66.

3. Perrotta FM, Marchesoni A, Lubrano E. Minimal disease activity and remission in psoriatic arthritis patients treated with anti-TNF- $\alpha$ drugs. J Rheumatol 2016;43:350-5.

4. Lubrano E, Parsons WJ, Perrotta FM. Assessment of response to treatment, remission, and minimal disease activity in axial psoriatic arthritis treated with tumor necrosis factor inhibitors. J Rheumatol 2016;43:918-23.

5. Perrotta FM, Lubrano E. Subcutaneous anti-TNF alfa induced sustained minimal disease activity and remission in psoriatic arthritis patients: a retrospective study. Postgrad Med 2016;128:693-6.

6. Smolen JS, Schöls M, Braun J, Dougados M, FitzGerald O, Gladman DD et al. Treating axial spondyloarthritis and peripheral spondyloarthritis, especially psoriatic arthritis, to target: 2017 update of recommendations by an international task force. Ann Rheum Dis 2018;77:3-17.

7. Schoels MM, Aletaha D, Alasti F, Smolen JS. Disease activity in psoriatic arthritis (PsA): defining remission and treatment success using the DAPSA score. Ann Rheum Dis 2016;75:811-8.
8. Coates LC, Fransen J, Helliwell PS. Defining disease activity in psoriatic arthritis: a proposed objective target for treatment. Ann Rheum Dis 2010;69:48-53.

9. Coates L, Helliwell P. Validation of minimal disease activity criteria for psoriatic arthritis using interventional trial data. Arthritis Car Res 2010;62:965-9.

10. Coates LC, Cook R, Lee KA, Chandran V, Gladman DD. Frequency, predictors, and prognosis of sustained minimal disease activity in an observational psoriatic arthritis cohort. Arthritis Care Res 2010;62:970-6.

11. Coates LC, Helliwell PS. Defining low disease activity states in psoriatic arthritis using novel composite disease instruments. J Rheumatol 2016;43:371-5.

12. Coates LC, Rahman P, Psaradellis E, Rampakakis E, Osborne B, Lehman AJ, et al. Validation of new potential targets for remission and low disease activity in psoriatic arthritis in patients treated with golimumab. Rheumatology 2019;58:522-6.

13. Healy PJ, Helliwell PS. Measuring clinical enthesitis in psoriatic arthritis: assessment of existing measures and development of an instrument specific to psoriatic arthritis. Arthritis Rheum 2008;59:686-91.

14. Fredriksson T, Pettersson U. Severe psoriasis--oral therapy with a new retinoid. Dermatologica 1978;157:238-44.

15. Ranza R, Marchesoni A, Calori G, Bianchi G, Braga M, Canazza $S$, et al. The Italian version of the Functional Disability Index of the Health Assessment Questionnaire. A reliable instrument for multicenter studies on rheumatoid arthritis. Clin Exp Rheumatol 1993;11:123-8

16. Lubrano E, Perrotta FM, Parsons WJ, Marchesoni A. Patient's global assessment as an outcome measure for psoriatic arthritis in clinical practice: a surrogate for measuring low disease activity? J Rheumatol 2015;42:2332-8.

17. Lubrano E, De Socio A, Perrotta FM. Comparison of composite indices tailored for psoriatic arthritis treated with csDMARD and bDMARD: a cross-sectional analysis of a longitudinal cohort. J Rheumatol 2017;44:1159-64.

18. Coates LC, Lubrano E, Perrotta FM, Emery P, Conaghan PG, Helliwell PS. What should be the primary target of "treat to target" in psoriatic arthritis? J Rheumatol 2019;46:38-42.

19. Wells GA, Boers M, Shea B, Brooks PM, Simon LS, Strand CV, et al. Minimal disease activity for rheumatoid arthritis: a preliminary definition. J Rheumatol 2005;32:2016-24.

20. Kilic G, Kilic E, Nas K, Kamanlı A, Tekeoglu İ. Residual symptoms and disease burden among patients with psoriatic arthritis: is a new disease activity index required? Rheumatol Int 2019;39:73-81.

21. Lubrano E, Perrotta FM. Psoriatic arthritis: is it time to treat-to-target or target to treat? Clin Rheumatol 2017;36:2633-5.

22. Lubrano E, Scriffignano S, De Socio A, Perrotta FM. Analysis of potential determinants for a treat-to-target strategy in psoriatic arthritis patients from a real-world setting. Clin Exp Rheumatol 2019;37:512.

23. van Mens LJ, Turina MC, van de Sande MG, Nurmohamed MT, van Kuijk AW, Baeten DL. Residual disease activity in psoriatic arthritis: discordance between the rheumatologist's opinion and minimal disease activity measurement. Rheumatology 2018; 57:283-90. 